\title{
Middle and posterior cardiac veins: An underused option for ventricular pacing
}

\author{
Henry M. Spotnitz, MD, ${ }^{\mathrm{a}}$ and Daniel Y. Wang, MD, ${ }^{\mathrm{b}}$ New York, NY
}

The middle and posterior cardiac veins are an alternative to the coronary sinus for left ventricular lead insertion, one that is well recognized by advocates of cardiac resynchronization therapy. ${ }^{1}$ These veins have not, however, been well described as a realistic alternative to thoracotomy for patients in whom leads cannot be passed across the tricuspid annulus. ${ }^{2,3}$ This communication describes 2 cases in which the posterior endocardial approach was used successfully, thus avoiding possible morbidity related to repeated thoracotomy in patients with complex disease and multiple previous cardiac operations.

\section{CLINICAL SUMMARIES}

\section{Patient 1}

A 31-year-old man with sinoatrial node dysfunction and third-degree heart block was referred for epicardial pacemaker insertion. The patient had previously undergone closure of atrial and ventricular septal defects as well as a maze procedure. Pulmonary and tricuspid valve replacements had each been done twice, most recently with a mechanical tricuspid valve. Both pleural spaces had been entered, and the patient had undergone reoperation for bleeding on at least 1 occasion. His history was otherwise notable for possible von Willebrand disease. He had anticoagulation with warfarin sodium (INN warfarin), a regimen that was converted to unfractionated heparin before the procedure. On clinical grounds, a transvenous approach was attempted initially. Venous access was achieved by left cephalic cutdown, and a Guidant Fineline II model 4471 positive fixation bipolar lead (Boston Scientific Corporation, Natick, Mass) was passed centrally through the innominate

\footnotetext{
From the Departments of Surgery ${ }^{\mathrm{a}}$ and Medicine, ${ }^{\mathrm{b}}$ Columbia University, New York, NY.

H.M.S. is the George H. Humphreys II Professor of Surgery at Columbia University and the Principal Investigator on the National Institutes of Health RO1 Grant HL080152, "Biventricular Pacing After Cardiopulmonary Bypass." D.Y.W. was supported by National Institutes of Health Training Grant T32 HL007854.

Disclosures: H.M.S. is a member of the Scientific Advisory Board of Biophan Technologies, Inc, Pittsford, NY. Authors have nothing else to disclose with regard to commercial support.

Received for publication Sept 28, 2011; revisions received Oct 18, 2011; accepted for publication Oct 26, 2011; available ahead of print Dec 12, 2011.

Address for reprints: Henry M. Spotnitz, MD, Department of Surgery, Columbia University Medical Center, 622 W 168th Street, Vanderbilt Clinic, 10th Floor, Rm 1010, New York City, NY 10032 (E-mail: hms2@columbia.edu).

J Thorac Cardiovasc Surg 2012;143:1223-5

$0022-5223 / \$ 36.00$

Copyright (c) 2012 by The American Association for Thoracic Surgery

doi:10.1016/j.jtcvs.2011.10.083
}

vein. A posterior vein was directly cannulated without a sheath, and the lead was passed distally as far as possible. The pacing threshold was $2.5 \mathrm{~V}$ at a pulse width of 0.5 milliseconds, an impedance of $1180 \Omega$, and an $\mathrm{R}$ wave of 12.0 $\mathrm{mV}$. There was an early increase in the pacing threshold, but at 6 months of follow-up the system was working well, with a ventricular pacing threshold of $2.3 \mathrm{~V}$ (Figure 1).

\section{Patient 2}

A 49-year-old woman was referred from an outside hospital for treatment of congestive heart failure with severe pulmonary hypertension. She had previously undergone 5 heart operations, including tricuspid valve replacement with a bioprosthesis on 3 occasions and an epicardial biventricular pacing system for heart block, supraventricular tachycardia, and heart failure. Details of the previous pacing systems were not available. The patient reported that her symptoms had worsened since her last heart operation, and her pacemaker was not functioning well. Problems included marked exit block on both leads and an exacerbation of congestive heart failure. Clinically, it was difficult to be certain whether the patient's heart failure reflected progressive tricuspid valve dysfunction or the loss of effective biventricular pacing. The patient's right ventricular systolic function, as assessed by 2-dimensional echocardiography, was severely reduced. She had anticoagulation with warfarin sodium, a regimen that was converted to enoxaparin sodium (INN enoxaparin), and was referred for epicardial lead revision. Tricuspid valvuloplasty was being considered, making the standard endocardial lead insertion unacceptable. We proposed endocardial coronary sinus lead insertion, tunneling the new lead to her abdominal pocket. Venous access was obtained initially through a left cephalic cutdown and subsequently by left subclavian puncture. The orifice of the coronary sinus was cannulated with a preformed coronary sinus cannula; however, there were no suitable lateral branches for pacing located on the venogram. A suitable site was finally located in a posterior vein, where the thresholds were low and diaphragmatic pacing could be avoided. The lead used was an $88-\mathrm{cm}$ Medtronic bipolar over-the-wire left heart lead (model 4194; Medtronic, Inc, Minneapolis, Minn). The pacing threshold was $0.6 \mathrm{~V}$ at a pulse width of 0.5 milliseconds, an impedance of $851 \Omega$, and an $\mathrm{R}$ wave of $26.8 \mathrm{mV}$. The highest threshold epicardial lead was capped, and the remaining ventricular lead (threshold 5.3 V) was connected 


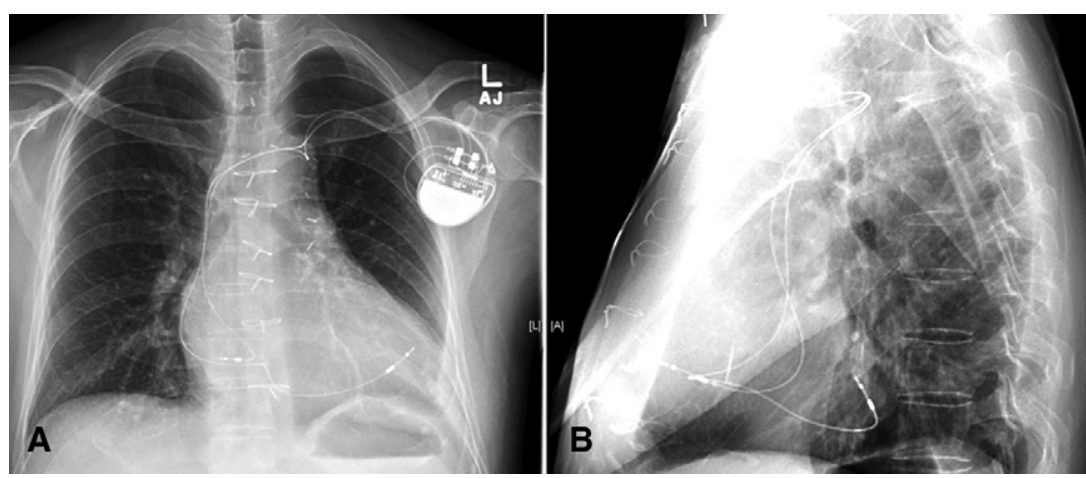

FIGURE 1. Posteroanterior (A) and lateral (B) radiographs of patient 1 with DDD pacemaker, including ventricular pacing with a bipolar lead in a posterior cardiac vein. This patient had previously undergone 3 cardiac operations, including tricuspid valve replacement with a mechanical prosthesis.

to the right ventricular lead port of the generator with a small safety margin to prolong battery life. The patient has done well during the ensuing year. The thresholds on the posterior lead remain at $1 \mathrm{~V}$, her congestive heart failure has improved, and right ventricular systolic function has normalized according to echocardiography (Figure 2).

\section{DISCUSSION}

Coronary sinus lead insertion as an alternative to thoracotomy for permanent pacing has been reported in previous case series ${ }^{4}$ but the use of posterior veins is less well described. ${ }^{2,3}$ Posterior veins are easier to access than the true coronary sinus and its lateral branches, which require acute cephalad angulation at the orifice. Among the risks of the endocardial approach in the presence of a mechanical tricuspid prosthesis is possible mechanical lead entrapment, a serious complication if the lead cannot be withdrawn. The small risk of entrapment must therefore be weighed against the risks of reoperative thoracotomy. Prosthetic lead rings project above the tricuspid annulus into the right atrium, tending to deflect an advancing lead peripherally toward the coronary sinus orifice. Other novel approaches to pacing access used to avoid formal thoracotomy include right parasternal mediastinotomy, transhepatic insertion, robotic approaches, and limited thoracotomy with special screw-in lead applicators. Concern exists that coronary sinus lead insertion could lead to thrombosis of the coronary sinus, but this is apparently rare. ${ }^{1}$ The fact that both patients had undergone previous thoracotomy provided some security if the fixed screw option was needed, as in the case of patient 1 . Lead advancement was also done without previous venography in the first case. Venography, customized insertion sheaths, and over-the-wire techniques can greatly facilitate coronary sinus lead insertion and are the current state of the art for primary coronary sinus lead insertion.

The radiographs presented here do not clearly demonstrate whether the caudal leads used are in the middle cardiac (posterior interventricular) vein or in a posterior left ventricular vein. The middle cardiac vein originates close to the orifice of the coronary sinus and follows the posterior interventricular groove toward the apex, whereas posterior veins originate on the posterior aspect of the left ventricle and drain into the coronary sinus further from
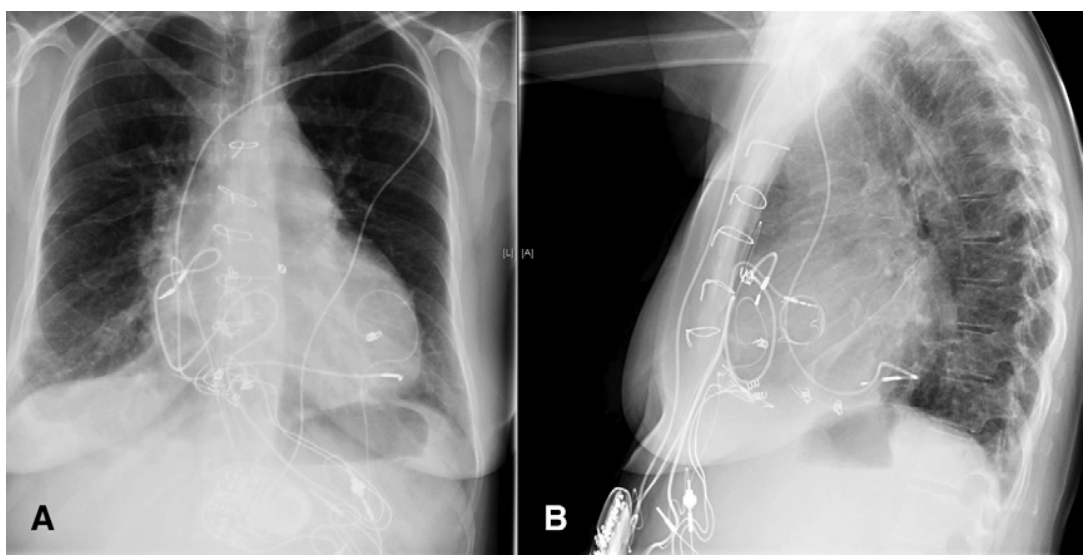

FIGURE 2. Posteroanterior (A) and lateral (B) radiographs of patient 2 with biventricular pacemaker, including ventricular pacing with a bipolar lead in a posterior or middle cardiac vein. This patient had previously undergone 5 cardiac operations, including 3 tricuspid valve replacements with a bioprosthesis. 
the orifice. ${ }^{5}$ Posterior veins are also referred to as inferolateral collaterals and left posterior veins. The coronary sinus venograms obtained during the treatment of patient 2 were not preserved. Echocardiograms demonstrate the lead entering the coronary sinus but do not define the final venous location. It is likely that the vein used in the treatment of patient 1 was a posterior vein, whereas the vein used in the treatment of patient 2 was a posterior or middle cardiac vein. Because the primary objective of lead insertion in these patients was ventricular pacing and not cardiac resynchronization therapy, the precise location of the veins involved is primarily of academic interest.

\section{CONCLUSIONS}

The middle and posterior cardiac veins provide access for ventricular pacing as an alternative to thoracotomy when passing a lead across the tricuspid annulus is contraindicated.
Access through these veins should be considered in patients with complicated surgical heart disease that includes a history of previous thoracotomy.

\section{References}

1. Giraldi F, Cattadori G, Roberto M, Carbucicchio C, Pepi M, Ballerini G, et al. Long-term effectiveness of cardiac resynchronization therapy in heart failure patients with unfavorable cardiac veins anatomy comparison of surgical versus hemodynamic procedure. J Am Coll Cardiol. 2011;58:483-90.

2. Goldstein DJ, Rabkin DG, Spotnitz HM. Unconventional approaches to cardiac pacing in patients with inaccessible cardiac chambers. Ann Thorac Surg. 1999; 67:952-8.

3. Nguyen LS, Swaroop S, Prejean CA. Pacing in the middle cardiac vein in a patient with tricuspid prosthesis. Pacing Clin Electrophysiol. 2002;25:243-4.

4. Hansky B, Güldner H, Vogt J, Minami K, Tenderich G, Horstkotte D, et al Coronary vein leads for cardiac pacing in patients with tricuspid valve replacement. Thorac Cardiovasc Surg. 2002;50:120-1.

5. Meisel E, Pfeiffer D, Engelmann L, Tebbenjohanns J, Schubert B, Hahn S, et al. Investigation of coronary venous anatomy by retrograde venography in patients with malignant ventricular tachycardia. Circulation. 2001;104: 442-7.

\title{
Early experience with the transaortic approach for transcatheter aortic valve implantation
}

\author{
Gopal Soppa, MRCS, PhD, David Roy, MD, Stephen Brecker, FRCP, and Marjan Jahangiri, FRCS (CTh), \\ London, United Kingdom
}

Transcatheter aortic valve implantation (TAVI) for the treatment of severe aortic stenosis (AS) is an increasingly viable option for patients at high risk or with contraindications for surgical aortic valve replacement (SAVR). ${ }^{1} \mathrm{TAVI}$ improves survival and quality of life in high-risk patients with severe AS compared with medical therapy and is comparable with SAVR. ${ }^{1}$ The transfemoral (TF) approach is the most common access route for deploying balloonexpandable valves. Alternative access routes in patients with severe peripheral vasculopathy include the transapical (TA), ${ }^{2}$ transaxillary (TAx), and, recently, the transaortic (TAo) approaches. ${ }^{3}$ We describe our early experience with the TAo approach.

\footnotetext{
From the Department of Cardiothoracic Surgery and Cardiology, St George's Hospital, University of London, London, United Kingdom.

Disclosures: Authors have nothing to disclose with regard to commercial support.

Received for publication Sept 14, 2011; revisions received Oct 20, 2011; accepted for publication Oct 31, 2011; available ahead of print Dec 12, 2011.

Address for reprints: Marjan Jahangiri, FRCS (CTh), Department of Cardiothoracic Surgery, St George's Hospital, Blackshaw Rd, London SW17 0QT, United King-

dom (E-mail: Marjan.Jahangiri@stgeorges.nhs.uk).

J Thorac Cardiovasc Surg 2012;143:1225-7

$0022-5223 / \$ 36.00$

Copyright (c) 2012 by The American Association for Thoracic Surgery

doi:10.1016/j.jtcvs.2011.10.082
}

\section{CLINICAL SUMMARY}

Between February and August 2011, 12 patients underwent TAVI with the TAo approach. Patients at high risk for SAVR are referred to a multidisciplinary meeting, where some will be scheduled to undergo surgical intervention, TAVI, or only medical treatment. ${ }^{4}$ Our method of approach for TAVI in the last 3 years has been primarily the TF approach or, if this is not possible, the TAx or TA approaches. However, because of the well-established minimally invasive SAVR procedure through a limited sternotomy ${ }^{5}$ in our unit and some of the complications associated with the TF approach, as well as the unsuitability of some patients for a peripheral approach, we started using the TAo technique.

After achievement of general anesthesia, a temporary pacing wire is placed through the internal jugular vein. A limited J-sternotomy into the third intercostal space is sufficient because access to the right atrium is not required. The proximal aorta is exposed, and a "soft" point for aortic puncture is identified by means of palpation and use of a metal marker under fluoroscopic guidance (Figure 1, $A$ ). This point is at least $6 \mathrm{~cm}$ above and in direct line with the aortic valve $(\mathrm{AV}$; Figure $1, B)$. Two purse-string sutures with Teflon pledgets are placed, and aortic puncture is performed. After crossing the AV by using the Seldinger 